\title{
Front Matter: Volume 7694
}

, "Front Matter: Volume 7694," Proc. SPIE 7694, Ground/Air Multi-Sensor Interoperability, Integration, and Networking for Persistent ISR, 769401 (19 May 2010); doi: 10.1117/12.864063

SPIE Event: SPIE Defense, Security, and Sensing, 2010, Orlando, Florida, United SPIE. States 


\section{PROCEEDINGS OF SPIE}

\section{Ground/Air Multi-Sensor Interoperability, Integration, and Networking for Persistent ISR}

Michael A. Kolodny

Editor

6-9 April 2010

Orlando, Florida, United States

Sponsored and Published by

SPIE

Volume 7694 
The papers included in this volume were part of the technical conference cited on the cover and title page. Papers were selected and subject to review by the editors and conference program committee. Some conference presentations may not be available for publication. The papers published in these proceedings reflect the work and thoughts of the authors and are published herein as submitted. The publisher is not responsible for the validity of the information or for any outcomes resulting from reliance thereon.

Please use the following format to cite material from this book:

Author(s), "Title of Paper," in Ground/Air Multi-Sensor Interoperability, Integration, and Networking for Persistent ISR, edited by Michael A. Kolodny, Proceedings of SPIE Vol. 7694 (SPIE, Bellingham, WA, 2010) Article CID Number.

ISSN 0277-786X

ISBN 9780819481580

Published by

SPIE

P.O. Box 10, Bellingham, Washington 98227-0010 USA

Telephone +1 3606763290 (Pacific Time) · Fax +1 3606471445

SPIE.org

Copyright (C) 2010, Society of Photo-Optical Instrumentation Engineers

Copying of material in this book for internal or personal use, or for the internal or personal use of specific clients, beyond the fair use provisions granted by the U.S. Copyright Law is authorized by SPIE subject to payment of copying fees. The Transactional Reporting Service base fee for this volume is $\$ 18.00$ per article (or portion thereof), which should be paid directly to the Copyright Clearance Center (CCC), 222 Rosewood Drive, Danvers, MA 01923. Payment may also be made electronically through CCC Online at copyright.com. Other copying for republication, resale, advertising or promotion, or any form of systematic or multiple reproduction of any material in this book is prohibited except with permission in writing from the publisher. The CCC fee code is 0277-786X/10/\$18.00.

Printed in the United States of America.

Publication of record for individual papers is online in the SPIE Digital Library.

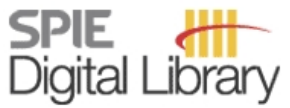

SPIEDigitalLibrary.org

Paper Numbering: Proceedings of SPIE follow an e-First publication model, with papers published first online and then in print and on CD-ROM. Papers are published as they are submitted and meet publication criteria. A unique, consistent, permanent citation identifier (CID) number is assigned to each article at the time of the first publication. Utilization of CIDs allows articles to be fully citable as soon they are published online, and connects the same identifier to all online, print, and electronic versions of the publication. SPIE uses a six-digit CID article numbering system in which:

- The first four digits correspond to the SPIE volume number.

- The last two digits indicate publication order within the volume using a Base 36 numbering system employing both numerals and letters. These two-number sets start with 00, 01, 02, 03, 04, $05,06,07,08,09,0 A, 0 B \ldots 0 Z$, followed by 10-1Z, 20-2Z, etc.

The CID number appears on each page of the manuscript. The complete citation is used on the first page, and an abbreviated version on subsequent pages. Numbers in the index correspond to the last two digits of the six-digit CID number. 


\section{Contents}

ix Conference Committee

xi Introduction

xiii Ground/air multisensor interoperability, integration, and networking for persistent ISR: what, why, who (7694-1)

M. A. Kolodny, U.S. Army Research Lab. (United States)

xV ARL Empire Challenge 2010 initiative (7694-17)

M. A. Kolodny U.S. Army Research Lab. (United States)

\section{SESSION 1 COALTION WARFARE I}

769404 Optimising the deployment of airborne heterogeneous sensors for persistent ISR missions [7694-03]

A. J. Barnett, G. Pearson, R. I. Young, Defence Science and Technology Lab. (United Kingdom)

769405 Aspects of sensor data fusion in interoperable ISR systems of systems for wide-area ground surveillance [7694-04]

W. Koch, M. Ulmke, J. Biermann, Fraunhofer FKIE (Germany); M. Sielemann, Federal Ministry of Defence (Germany)

769406 Distributed policy based access to networked heterogeneous ISR data sources [7694-05] G. Bent, D. Vyvyan, IBM United Kingdom Ltd. (United Kingdom); D. Wood, P. Zerfos, S. Calo, IBM Thomas J. Watson Research Ctr. (United States)

\section{SESSION 2 COALTION WARFARE II}

769409 Management of coalition sensor networks [7694-08]

D. C. Verma, IBM Thomas J. Watson Research Ctr. (United States); T. Brown, C. Ortega, The City Univ. of New York (United States)

7694 OA Airborne multisensor demonstrator program for persistent wide-area surveillance [7694-09] S. J. Rouse, R. I. Young, B. D. McGrath, Defence Science and Technology Lab. (United Kingdom)

\section{SESSION 3 PERSISTENT SURVEILLANCE}

7694 OB Generic Vehicle Architecture for the integration and sharing of in-vehicle and extra-vehicle sensors [7694-10]

F. Bergamaschi, D. Conway-Jones, IBM Hursley Lab. (United Kingdom); N. Peach, PB

Partnership Ltd. (United Kingdom) 
7694 OC Persistent surveillance using mutually-visible robotic formations [7694-11]

E. A. Stump, B. M. Sadler, U.S. Army Research Lab. (United States)

7694 OD An automatic UAV search, intercept, and follow algorithm for persistent surveillance [7694-12]

G. E. Collins, Toyon Research Corp. (United States)

$7694 \mathrm{OE} \quad$ User evaluation of a GUI for controlling an autonomous persistent surveillance team [7694-13]

P. Scerri, S. Owens, K. Sycara, Carnegie Mellon Univ. (United States); M. Lewis, Univ. of Pittsburgh (United States)

7694 OF Environmental awareness for sensor and emitter employment [7694-14]

K. K. Yamamoto, D. K. Wilson, U.S. Army Cold Regions Research and Engineering Lab. (United States)

\section{SESSION 4 INTEROPERABILITY I}

7694 OK CityBeat @ Tec^Edge [7694-19]

J. D. Graley, L. E. Quinn, Air Force Research Lab. (United States); A. P. Palomino, Intelligent Software Solutions, Inc. (United States)

$7694 \mathrm{OL} \quad$ Unattended ground sensors standards working group (Keynote Paper) [7694-20] R. Heathcock, Defense Intelligence Agency (United States); K. Linnebur, C. Brasch, MITRE Corp. (United States)

$76940 \mathrm{M} \quad$ Standards and protocols for interoperation of unattended ground sensors [7694-21] G. H. Stolovy, U.S. Army Research Lab. (United States)

7694 ON A roadmap for future UGS [7694-22]

J. H. McQuiddy, McQ, Inc. (United States)

$769400 \quad$ Interoperability of unattended ground sensors with an open architecture controller using SensorML [7694-23]

J. Chambers, S. Fairgrieve, Northrop Grumman Corp. (United States)

\section{SESSION 5 INTEROPERABILITY II}

7694 OR Open-source-based architecture for layered sensing applications [7694-26]

D. A. Uppenkamp, T. V. Rovito, K. L. Priddy, Air Force Research Lab. (United States)

7694 OS Collaborative air/ground command and control for responsive persistent ISR operations using unmanned systems [7694-27]

R. Ordower, L. Dixon, SAIC (United States); N. Lynch, U.S. Army Communications-Electronics Research, Development and Engineering Ctr. (United States) 
7694 OT Heterogeneous sensor networks: a bio-inspired overlay architecture [7694-28]

J. Burman, Teledyne Scientific Co. (United States); J. Hespanha, U. Madhow, D. Klein, J. Isaacs, S. Venkateswaran, Univ. of California, Santa Barbara (United States); T. Pham, U.S. Army Research Lab. (United States)

7694 OU Optimal placement of multiple types of communicating sensors with availability and coverage redundancy constraints [7694-29]

S. N. Vecherin, U.S. Army Cold Regions Research and Engineering Lab. (United States) and New Mexico State Univ. (United States); D. K. Wilson, U.S. Army Cold Regions Research and Engineering Lab. (United States); C. L. Pettit, U.S. Naval Academy (United States)

7694 OV Unmanned vehicle technology for networked non-line-of-sight sensing applications [7694-30]

M. Gates, Louisiana Tech Univ. (United States); G. Pepper, Tennessee State Univ. (United

States); A. K. Mitra, C. Hu, Air Force Research Lab. (United States); S. Zein-Sabatto, T. Rogers, Tennessee State Univ. (United States); R. Selmic, Louisiana Tech Univ. (United States);

E. Hamdan, North Carolina A\&T State Univ. (United States); M. Malkani, Tennessee State Univ. (United States)

7694 OW High-performance, miniature RF transceivers for energy-aware UGSS [7694-31]

M. E. Barr, L. A. Webster, D. Maldonado, L-3 Communications Nova Engineering (United States)

7694 OX Network of acoustic sensors for the detection of weapons firing: tests for the choice of individual sensing elements [7694-32]

P. Naz, French-German Research Institute of Saint-Louis (France); Ch. Marty, La Délégation Générale pour l'Armement (France); S. Hengy, P. Hamery, French-German Research Institute of Saint-Louis (France)

7694 OY Mobile optical detection system for counter-surveillance [7694-33]

L. Sadler, T. A. Alexander, U.S. Army Research Lab. (United States)

$76940 Z$ Implementation of utility-based resource optimization protocols on ITA Sensor Fabric [7694-34]

S. Eswaran, Pennsylvania State Univ. (United States); A. Misra, Telcordia Technologies, Inc. (United States); F. Bergamaschi, IBM Hursley Lab. (United Kingdom); T. La Porta, Pennsylvania State Univ. (United States)

\section{SESSION 7 NEW TECHNOLOGY}

769410 Compact networked radars for Army unattended ground sensors [7694-35]

D. A. Wikner, E. A. Viveiros, U.S. Army Research Lab. (United States); R. Wellman, General Technical Services (United States); J. Clark, U.S. Army Research Lab. (United States); J. Kurtz, Univ. of Florida (United States); J. Pulskamp, U.S. Army Research Lab. (United States); R. Proie, T. Ivanov, General Technical Services (United States); R. G. Polcawich, E. D. Adler, U.S. Army Research Lab. (United States)

$769411 \quad$ Profiling system design tradeoffs using the sparse detector sensor model [7694-36] A. L. Robinson, C. E. Halford, The Univ. of Memphis (United States) 
769413 Profiling sensor classification algorithm implementation on an embedded controller [7694-38]

R. K. Reynolds, D. J. Russomanno, S. K. Chari, C. E. Halford, The Univ. of Memphis (United States)

769414 Mission specification and control for unmanned aerial and ground vehicles for indoor target discovery and tracking [7694-39]

P. D. Ulam, Z. Kira, R. C. Arkin, T. R. Collins, Georgia Institute of Technology (United States)

769415 The layered sensing operations center: a modeling and simulation approach to developing complex ISR networks [7694-40]

C. Curtis, M. Lenzo, M. McClure, B. Preiss, Air Force Research Lab. (United States)

769416 Pheromone-based coordination strategy to static sensors on the ground and unmanned aerial vehicles carried sensors [7694-41]

E. Pignaton de Freitas, Halmstad Univ. (Sweden) and Federal Univ. of Rio Grande do Sul (Brazil); T. Heimfarth, Federal Univ. of Lavras (Brazil); C. E. Pereira, Federal Univ. of Rio Grande do Sul (Brazil); A. Morado Ferreira, Military Insitute of Engineering (Brazil); F. Rech Wagner, Federal Univ. of Rio Grande do Sul (Brazil); T. Larsson, Halmstad Univ. (Sweden)

769419 Real-time geo-registered steerable video generation [7694-44]

P. Maenner, S. L. Dockstader, R. Shuler, B. V. Brower, M. F. Pellechia, ITT Geospatial Systems (United States)

\section{SESSION 8 SENSOR, DATA, AND INFORMATION FUSION}

7694 IB Multi-asset control, sensor fusion, and information sharing through a centralized operator station [7694-46]

D. L. Gerlock, D. Buster, K. U. Schultz, Honeywell, Inc. (United States)

7694 1C Sensor fusion for ISR assets [7694-47]

T. Damarla, U.S. Army Research Lab. (United States)

7694 ID Services oriented architecture (SOA)-based persistent ISR simulation system [7694-48] G. Chen, DCM Research Resources, LLC (United States); E. Blasch, Air Force Research Lab. (United States); D. Shen, DCM Research Resources, LLC (United States); H. Chen, Univ. of New Orleans (United States); K. Pham, Air Force Research Lab. (United States)

7694 IE Flexible application for consolidation and presentation of intelligence surveillance, and reconnaissance data [7694-50]

D. R. Garrison II, Harris Corp. (United States)

POSTER SESSION

$76941 \mathrm{H} \quad$ Robust extended target detection using nonlinear morphological operations [7694-54] H.-W. Chen, C. Volpe, M. Tarnowski, S. Snarski, Applied Research Associates, Inc. (United States) 
7694 II Low-resolution vehicle tracking using dense and reduced local gradient features maps [7694-55]

M. P. Dessauer, S. Dua, Lovisiana Tech Univ. (United States)

$76941 \mathrm{~J}$ Optical flow object detection, motion estimation, and tracking on moving vehicles using wavelet decompositions [7694-56]

M. P. Dessauer, S. Dua, Lovisiana Tech Univ. (United States)

7694 IK Range and velocity independent classification of humans and animals using a profiling sensor [7694-57]

S. Chari, F. Smith, C. Halford, E. Jacobs, J. Brooks, The Univ. of Memphis (United States)

Author Index 
Downloaded From: https://www.spiedigitallibrary.org/conference-proceedings-of-spie on 26 Apr 2023

Terms of Use: https://www.spiedigitallibrary.org/terms-of-use 


\title{
Conference Committee
}

\author{
Symposium Chair
}

Michael T. Eismann, Air Force Research Laboratory (United States)

Symposium Cochair

William Jeffrey, HRL Laboratory, LLC (United States)

Conference Chair

Michael A. Kolodny, U.S. Army Research Laboratory (United States)

Conference Cochairs

Tien Pham, U.S. Army Research Laboratory (United States)

Kevin L. Priddy, U.S. Army Research Laboratory (United States)

Program Committee

Jacques Bédard, Defence Research and Development Canada Valcartier (Canada)

Robert Heathcock, Defense Intelligence Agency (United States)

Jeff Houser, U.S. Army Research Laboratory (United States)

Gavin Pearson, Defence Science and Technology Laboratory (United Kingdom)

Stephen G. Perry, MTC Services Corp (United States)

Ronald B. Sartain, U.S. Army Research Laboratory (United States)

King Siu, U.S. Army Armament Research, Development and Engineering Center (United States)

Raja Suresh, General Dynamics Advanced Information Systems (United States)

Graeme P. van Voorthuijsen, TNO Defence, Security and Safety (Netherlands)

Rob Williams, Air Force Research Laboratory (United States)

\section{Session Chairs}

1 Coaltion Warfare I

Michael A. Kolodny, U.S. Army Research Laboratory (United States)

Tien Pham, U.S. Army Research Laboratory (United States) 
Coaltion Warfare II

Gavin Pearson, Defence Science and Technology Laboratory

(United Kingdom)

Michael A. Kolodny, U.S. Army Research Laboratory (United States)

3 Persistent Surveillance

Jacques Bédard, Defence Research and Development Canada

(Canada)

Graeme P. van Voorthuijsen, TNO Defence, Security and Safety

(Netherlands)

$4 \quad$ Interoperability I

Rob Williams, Air Force Research Laboratory (United States)

Jeff Houser, U.S. Army Research Laboratory (United States)

5 Interoperability II

Tien Pham, U.S. Army Research Laboratory (United States)

Rob Williams, Air Force Research Laboratory (United States)

6 Sensor Networks and Communications: Joint Session with Conference 7707

Michael A. Kolodny, U.S. Army Research Laboratory (United States)

$7 \quad$ New Technology

King Siu, U.S. Army Armament Research, Development and Engineering Center (United States)

Ronald B. Sartain, U.S. Army Research Laboratory (United States)

8 Sensor, Data, and Information Fusion

Graeme P. van Voorthuijsen, TNO Defence, Security and Safety (Netherlands)

Michael A. Kolodny, U.S. Army Research Laboratory (United States) 


\section{Introduction}

This was a new conference that was introduced at the SPIE Defense, Security, and Sensing symposium held in Orlando, Florida. Conference 7694 was Ground/Air Multi-Sensor Interoperability, Integration, and Networking for Persistent ISR and ran 6-9 April 2010. The goal of this conference is to bring together the technical, operational (users) and policy community to provide a forum for discussion of problems, issues and technology involving interoperability for persistent ISR. The conference had about 50 technical papers in oral presentations and another 10 papers presented during the poster session. The conference was highlighted by four keynote speakers, who are centered in the world of ISR interoperability at the coalition warfare level, and an open forum panel in which there was a "no holds barred" audience questioning of the panel members. I hope you find the proceedings both provocative and informative.

I am looking forward to an even more exciting conference at SPIE Defense, Security, and Sensing in 2011, and hope that everyone will join us.

Michael A. Kolodny 
Downloaded From: https://www.spiedigitallibrary.org/conference-proceedings-of-spie on 26 Apr 2023

Terms of Use: https://www.spiedigitallibrary.org/terms-of-use 